\title{
Simulation of a Mechanical Thrombectomy Device Based in the Use of Self- Expandable Stents for the Blood Clots Extraction.
}

\author{
G. Romero, I. Moreno, M.L. Martinez \\ ETSI Engineering, Universidad Politecnica de Madrid \\ Madrid, Spain \\ e-mail: gregorio.romero@upm.es, \\ isabel.moreno@upm.es, luisa.mtzmuneta@upm.es
}

\author{
G. Pearce \\ Keele University \\ Keele, United Kingdom \\ e-mail: gpearce2011@googlemail.com
}

\begin{abstract}
Recently, we have presented some studies concerning the analysis, design and optimization of one experimental device developed in the UK - GPTAD - which has been designed to remove blood clots without the need to make contact with the clot itself, thereby potentially reducing the risk of problems such as downstream embolisation. Based on the idea of a modification of the previous device, in this work, we present a model based in the use of stents like the SolitaireTM FR, which is in contact with the clot itself. In the case of such devices, the stent is self-expandable and the extraction of the blood clot is faciliatated by the stent, which must be inside the clot. Such stents are generally inserted in position by using the guidewire inserted into the catheter. This type of modeling could potentially be useful in showing how the blood clot is moved by the various different forces involved. The modelling has been undertaken by analyzing the resistances, compliances and inertances effects. We model an artery and blood clot for range of forces for the guidewire. In each case we determine the interaction between blood clot, stent and artery.
\end{abstract}

Keywords-Biomedical engineering;Stent; Thrombectomy Device; Simulation techniques.

\section{INTRODUCTION}

The World Health Organization reports that 15 million people worldwide suffer stroke; and of these, 5 million die and a further 5 million are left permanently disabled, many severely impaired. Consequently stroke is a major cause of mortality world-wide. Most strokes are caused by a blood clot that occludes an artery in the cerebral circulation. Thrombolytic agents such as Alteplase are used to dissolve blood clots that arise in the cerebral arteries of the brain but there are limitations on the use. Recently screening for patients at risk of strokes and TIA's has come into being. If such plaques are detected in the carotid arteries (by Ultrasound), a Carotid endarterectomy (CEA) - a surgical operation - may be performed to remove the occlusive plaque. Additionally, stenting has been investigated for partially occluded carotid arteries. In the SAPPHIRE study [1], it was concluded that carotid stenting lowered the incidences of major stroke and myocardial infarction, in patients at high risk who needed surgery. Over the past decade, other methods of treatment have been developed which include Thrombectomy Devices. Such devices have the potential to be used as an alternative to thrombolytic agents or in conjunction with them to extract clots in the different arteries e.g. in the middle cerebral artery of the brain, carotid, popliteal artery, etc.

Alternatively a clot of blood may become attached to the plaque and subsequently become detached and pass into the cerebral circulation giving rise to a stroke. In the case of $100 \%$ occlusion, it causes total blockage of the artery. In this work, we present an analysis and modelling a 100\% occlusion case and without any existence of plaque. To model this case we take into account factors such as the resistances, compliances and inertances effects associated with the blood clot and the stent in the modelling. Such modelling will be useful in optimizing the velocity of the cable and predicting the result of clot extraction under a variety of conditions. The model includes the systolic and diastolic blood pressure variations. The aim of this simulation model is to obtain a range of velocities appropriated to the use of these types of mechanical thrombectomy devices.

\section{MODEL DESCRIPTION}

In recent times the mechanical thrombectomy devices have become increasingly involved in blood clot removal. The SolitaireTM FR stent is a fully recoverable, selfexpanding thrombectomy device that is based on the Solitaire/Solo stent. In the process, of clot extraction a microcatheter is tracked over a guidewire to the point of the blockage. The guidewire is then retracted and the Solitaire ${ }^{\mathrm{TM}}$ FR system is advanced through the microcatheter until the entire length of the Solitaire FR stent is distal to the blockage. The balloon is then inflated to occlude antegrade blood flow. The Solitaire FR is then deployed until the distal marker of the microcatheter is aligned with the proximal marker of the stent (fig. 1.A and 1.B). The deployed stent and microcatheter are then retracted simultaneously as one unit through the balloon guide catheter while constant aspiration is performed on the balloon guide catheter with a syringe (fig. 1.C) [2].

Comparing with GP Thrombus Aspiration Device (GPTAD), the Solitaire ${ }^{\mathrm{TM}}$ FR needs to touch the clot to remove it. Touching a clot in any way may potentially cause a risk of downstream embolisation. The use of self expanding stents may cause less endothelial damage compared to balloon-expandable stents, which may lower rates of re-stenosis 


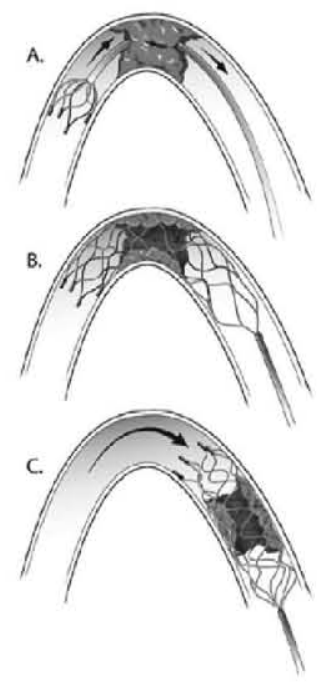

Figure 1. Solitaire FR Thrombectomy Procedure. [2]

Although the full procedure involves inserting a balloon guide catheter and tracking a microcatheter in addition to the stent, the model shown here only analyzes the interaction blood clot-stent-artery.

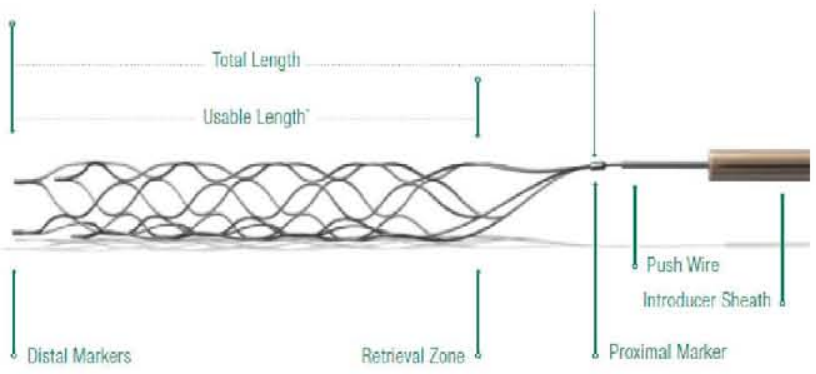

Figure 2. Solitaire Stent detail [3]

This device is introduced into the artery at the place where the clot is situated, and is positioned into the blood clot. Once the stent has been inserted into the blood clot it self-expandes and a guidewire provides the necessary force for the extraction. The clot is then manually extracted from the body.

\section{BLOOD CLOT AND FRICTION MODELLING}

The objective of this study is to introduce a new model that can be used to investigate and assist the final design of a device that incorporates the use of stents. We investigate the potential performance of such a device under different conditions of blood flow, size of blood clot and systolicdiastolic pressure, in a given vessel. The method chosen for the representation and simulation in this instance is the Bond Graph technique [4][5], which allows assimilating the model to an electric circuit made up of inductances, capacitances and resistances. Therefore, it is possible to obtain the results in a simple way by evaluating flows and efforts that join and connect the components of the model.
Previous models developed by the authors concerning the 'GPTAD' device [6][7][8] have shown good results but some simplifications concerning the interaction inside the blood clot and with the artery wall only were considered.

To develop the modeling further it is necessary to analyze the inertances, the compressibility that the artery are subjected to, and the resistances [9]. In addition, the friction between the clot and the arterial wall creates another resistance factor. The value of this parameter must be variable depending on whether the clot has begun its movement (dynamic friction) or before it has begun to move (static friction) during the clot extraction procedure. When the clot begins to move the friction decreases considerably. In the previous developed models it was obtained only from the Stokes equation; in the work presented in this paper, this value is obtained from the platelet interaction - artery wall for the static friction and with Stokes for the dynamic friction; the transition between both values marks the beginning or the end of the clot movement.

Finally, into the model developed and presented here we have included the systolic and diastolic blood pressure $(120 / 80 \mathrm{mmHg})$ as additional positive pressure adding a variable pressure source that pushes the clot in synchrony with the rhythm of the heart.

Accurately defining the clot model in order to model it is the most complex part of the modelling. In previous models the clot has been approximated to a cylindrically-shaped element of [0.5-5] cm long, and of a mass that falls between [0.5-0.1] gram, connected to the artery wall by using an equivalent spring. Using this equivalent spring technique in the modelling enables us to determine when clot movement begins. We calculate the displacement of the spring when it is subjected to $0.01 \mathrm{~N}$ (and other values depending of various parameters) via a typical spring equation. Therefore, only when the spring underwent this displacement does the clot begin to move; no clot movement occurs before this value is achieved.

The interaction between the blood clot and the artery of used in the previous models can be developed further here in this model. Previous models focused on the simulated adhesion of the clot to the wall. The existing model considers the clot as one cylindrical shape with the same adhesion force in every case and a spring-damper systems in parallel, which simulates the elastoplastic ability of the clot to withstand traction. This representation of the internal conditions of the clot will be maintained in all subsequent models, although they change their name. Additionally, the clot inertia also involves the bond strength to the artery.

Modelling the adhesion force itself is complex. It is relatively difficult to find the spring-damper rate that represents the junction with the arterial wall and previously - only the in-vitro maximum adhesion force was considered. Secondly, the junction with the wall had to allow, the clot to move, even if the suction pressure applied was insufficient to move it in reality. The value of the constants in both the spring and the damper must be extremely high to simulate a firm anchor to the point of release. Simulating the moment when the clot breaks loose from the wall in the previous model method was very challenging. 
Due to these problems with the previous model we sought another solution to simulate the clot and its behavior under pressure. As in the previous modelling, we kept the partitions represented by inertia and joined by spring-damper system $\left(R_{\text {union, }} K_{\text {union }}\right)$, to simulate the elastic and plastic behavior of the clot.

To model the junction with the artery, the point of release and the static and dynamic friction we decided to add to each inertia an effort source $\left(\mathrm{F}_{\mathrm{adh}}\right)$ that varies depending of the force applied over each blood clot part when the simulation is running. We can observe the new configuration in figure 3 .

When the clot begins its movement, the static friction disappears and the dynamic friction acts in the system. It is much lower that the static friction. We have calculated it by means of the Stokes law for a cylindrical solid:

$$
F_{\text {dynamic_friction }_{-}}=\frac{C}{8} \cdot \rho \cdot \pi \cdot D^{2} \cdot V^{2}
$$

where ' $\mathrm{C}$ ' is the form coefficient for a cylinder, ' $\mathrm{p}$ ' is the blood's density, 'D' the clot's diameter and ' $V$ ' the velocity of the first partition.

Each inertia will suffer a force due to suction, which should be compensated in the model with a force of friction to annul it, while the clot is in the position of static friction. Once we have calculated the flow-effort table of the system, we apply the condition that the stress on the inertia must be zero. The condition to know if the clot is attached to the surface is based on the forces that the springs encounter between partitions $\left(\mathrm{K}_{\text {union }}\right)$.

Hence when the effort supported by each partition is higher than the adherence force then the clot part releases from the surface. The value of the adherence force was analyzed by C. J. Flannery (2005) [10] and we use all the necessary data from it.

However in calculating the adhesion strength C.J Flannery did not consider cylindrical clot geometry that narrows down the middle due to stenosis or atherosclerotic plaque. In our modelling we consider the non existence of a plaque, and consider the blood clot as a cylinder divided in five parts in order to study better the interaction with the wall artery.

As we have defined our clot we calculated the adhesion force by means of the platelet adhesion force. And we have obtained from C.J. Flannery the next equation:

$$
\mathrm{N}^{\circ} \text { platelets per area }=f p \cdot \frac{S A}{M P A}
$$

where 'fp' is the \% of platelets in the clot, 'SA' is the surface in contact with the artery and 'MPA' the Mean Platelet Area.

Once we have the number of platelets in contact with the artery and the force/platelet, we can calculate the adhesion force for each partition and obviously of the entire clot. In this manner we would have different adhesion forces depending on the form and size of the clot.

$$
\mathrm{F}_{\text {adh }}=\mathrm{N}^{\mathrm{o}} \text { platelets }{ }_{\text {TотAL }} \cdot F_{\text {platelet }- \text { artery_wall }}
$$

The systolic and diastolic blood pressure can be introduced by using a variable pressure. If we consider that the blood pressure varies from about $120 \mathrm{mmHg}$ to $80 \mathrm{mmHg}$ $(16 \mathrm{kPa}$ to $11 \mathrm{kPa})$ in systolic to diastolic pressure variation in the normal cardiac cycle, and we impose a rate of 1 cycle per second, we can approximate mathematically the pressure $(\mathrm{kPa})$ in two parts $(0.00-0.32 \mathrm{sec}$. and $0.32-1.00 \mathrm{sec}$.) by using two polynomial expressions (4) and (5) on the following page.
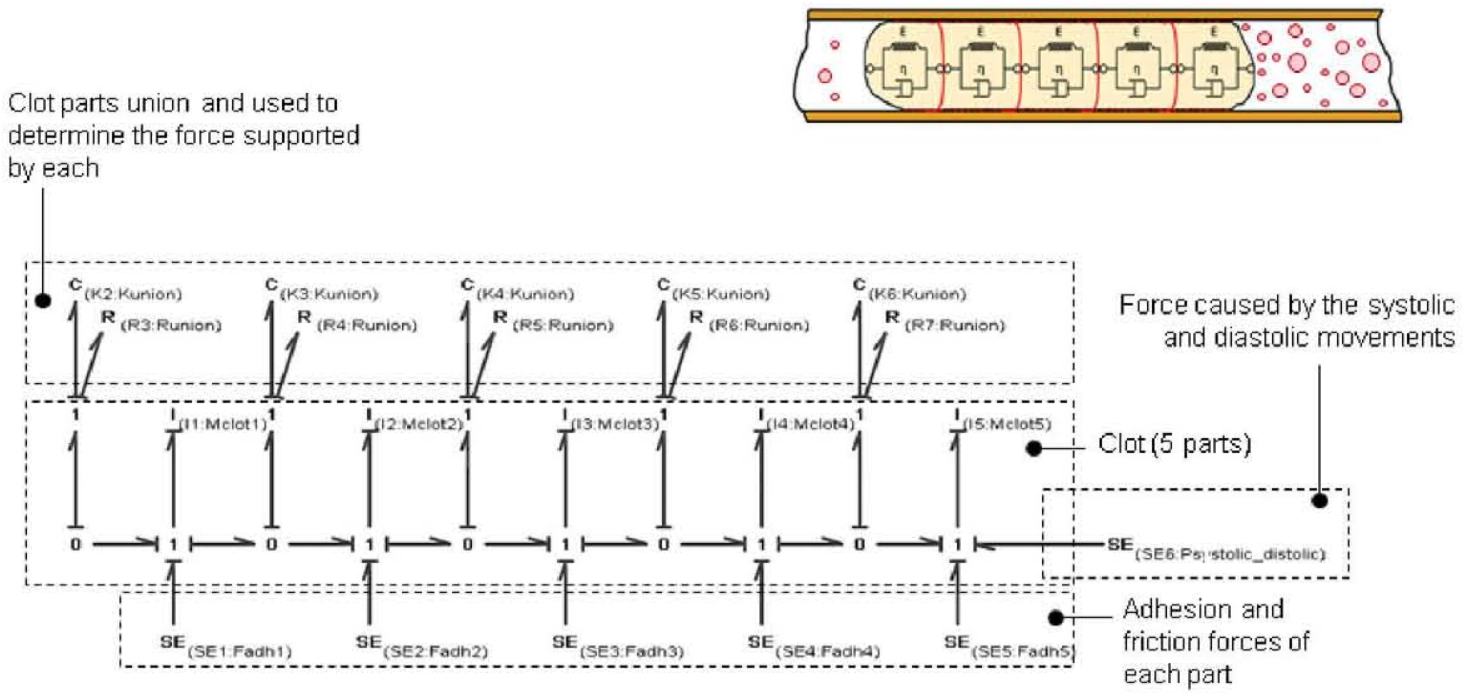

Figure 3. Model of blood clot-artery interaction 


$$
\begin{aligned}
& P_{a}=13415 \cdot t^{5}-8508,6 \cdot t^{4}+986,58 \cdot t^{3}+177,3 \cdot t^{2}-4,99 \cdot t+11,01 \\
& P_{b}=-1488 \cdot t^{6}+6237,6 \cdot t^{5}-10700 \cdot t^{4}+9595,3 \cdot t^{3}-4719,5 \cdot t^{2}+1188,3 \cdot t^{1}-102,8
\end{aligned}
$$

The previous equations have been obtained by taking different points from the typical pressure waveform associated.

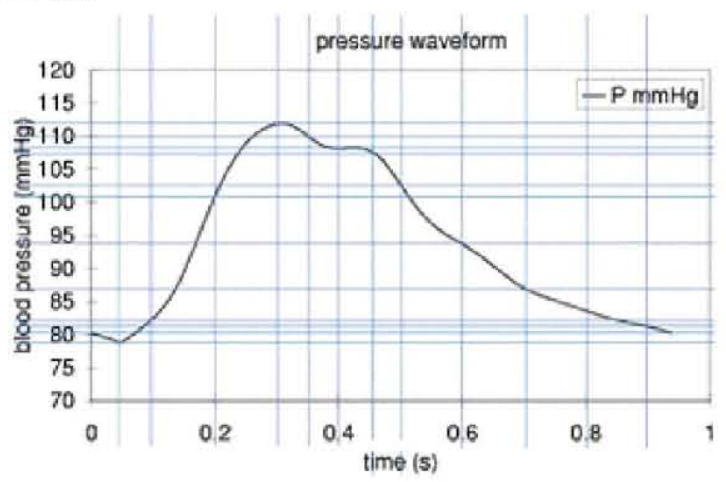

Figure 4. Systolic and diastolic blood pressure waveform

\section{STENT MODELLING}

To model the stent, it's necessary to take into account the environment inside the blood clot when the stent is depolyed, in relation to factors such as the elastic component in parallel configuration with the blood clot. In relation to the five parts model shown in figure 3 , we include the elastic stent components divided in five segments too. This technique permits us to differentiate when the clot is being separated from the artery wall or not.

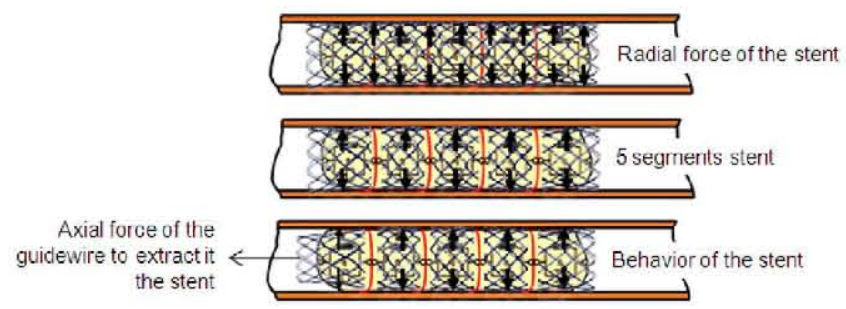

Figure 5. Five parts blood clot and stent scheme

It can be seen in figure 5 , that when the stent is open into the blood clot, the stent expands automatically and compression to the artery wall occurs in a radial direction, thus creating a radial force. The clot is then pulled by using the guidewire, and an axial force appears being applied over the guidewire. This results in an increase in the length as for a typical spring; in this moment, the radial force decrease and the movement of the blood clot begins if the force that appear over it is greater than the force in the contact with the artery wall, i.e., the usual adhesion force plus the residual radial force of the stent.

Jedwab and Clerc [11] developed a mathematical model of a self-expanding metallic stent with the goal of computing several geometrical and mechanical properties of it, which is analyzed in the Conti work too [12]. The theoretical model is based on four main assumptions:

- the stent is a combination of a number of independent open-coiled helical springs undergoing large deformations;

- the extremities of the stent are not free to rotate due to the friction between the stent wires in the crossing points;

- the springs experience only elastic deformation;

- the springs have large ratio $\mathrm{D} / \mathrm{d}$, were ' $\mathrm{D}$ ' is the stent average diameter and ' $d$ ' is the stent wire diameter.

Under these conditions, they may use the equation for open-coiled helical springs given by Wahl [13]. Considering an axial force ' $\mathrm{F}$ ' acting on the stent, it leads to an elongation of the stent and a change in the pitch angle $(\beta)$.

So they obtained an analytical relation between the axial force and the initial and the current pitch angle after assigning the material properties. It can be written as an explicit function of the current pitch angle $(\beta)$ and the initial pitch angle $\left(\beta_{0}\right)$ :

$F=n\left[\frac{G I_{p} \cos \beta}{R}\left(\frac{2 \sin \beta \cos \beta}{R}-\frac{2 \sin \beta_{0} \cos \beta_{0}}{R_{0}}\right)-\frac{E I \sin \beta}{R}\left(\frac{2 \cos ^{2} \beta}{R}-\frac{2 \cos ^{2} \beta_{0}}{R_{0}}\right)\right](6)$

, where ' $n$ ' is the number of wires, ' $I$ ' and ' $I_{p}$ ' are the wire moment of inertia and polar moment of inertia, respectively, ' $\mathrm{E}$ ' is Young's modulus of elasticity, ' $\mathrm{G}$ ' the rigidity modulus, and ' $\mathrm{R}_{0}$ ' / ' $\mathrm{R}$ ' the initial and work stent radius respectively.

In a similar way, in the thesis developed by Loo [14], he obtain the equivalent longitudinal and radial compliance as function of ' $\mathrm{K}_{1}$ ', ' $\mathrm{K}_{2}$ ' and ' $\mathrm{K}_{3}$ ' parameters, which are constants based in the ' $\beta_{0}$ ' and ' $\mathrm{D}_{0}$ ' values.

$$
\begin{array}{r}
K_{L}=\frac{2 n}{K_{3} \pi c \cos \beta}\left[\frac{G I_{p}}{K_{3}}\left(\frac{2 \cos \beta}{K_{3}}\right)-\frac{E I}{K_{3}}\left(\frac{2 \cos \beta}{K_{3}}-K_{2} \sec ^{2} \beta\right)\right] \\
K_{r}=\frac{2 c}{K_{3} \sin \beta(D L \tan \beta)^{2}}\left[2 D L n \operatorname { t a n } \beta \left(\frac{G I_{p}}{K_{3}}\left(\frac{2 \cos \beta}{K_{3}}\right)-\right.\right. \\
\left.\left.-\frac{E I}{K_{3}}\left(\frac{2 \cos \beta}{K_{3}}-K_{2} \sec ^{2} \beta\right)\right)-F\left(\frac{D L}{\cos ^{2} \beta}+K_{3} \sin \beta(\pi c D-L \tan \beta)\right)\right]
\end{array}
$$

To implement this behaviour into the simplified model, will be necessary to introduce a longitudinal compliance and resistance (fig. 6), which will go in parallel with the springdumper system used to model the blood clot. Additionally, will be necessary to introduce a radial force, corresponding with the ' $\mathrm{K}_{\mathrm{r}}$ ' value multiplied by the radius reduction; the radius reduction will be given taking into account the initial pitch angle $\left(\beta_{0}\right)$ and the current pitch angle $(\beta)$.

In each step of simulation the ' $\mathrm{K}_{\mathrm{L}}$ ' and ' $\mathrm{K}_{\mathrm{r}}$ ' values must be recalculated from the current pitch angle in each moment; so the compliance stent values are not linear according with expressions (7) and (8). 


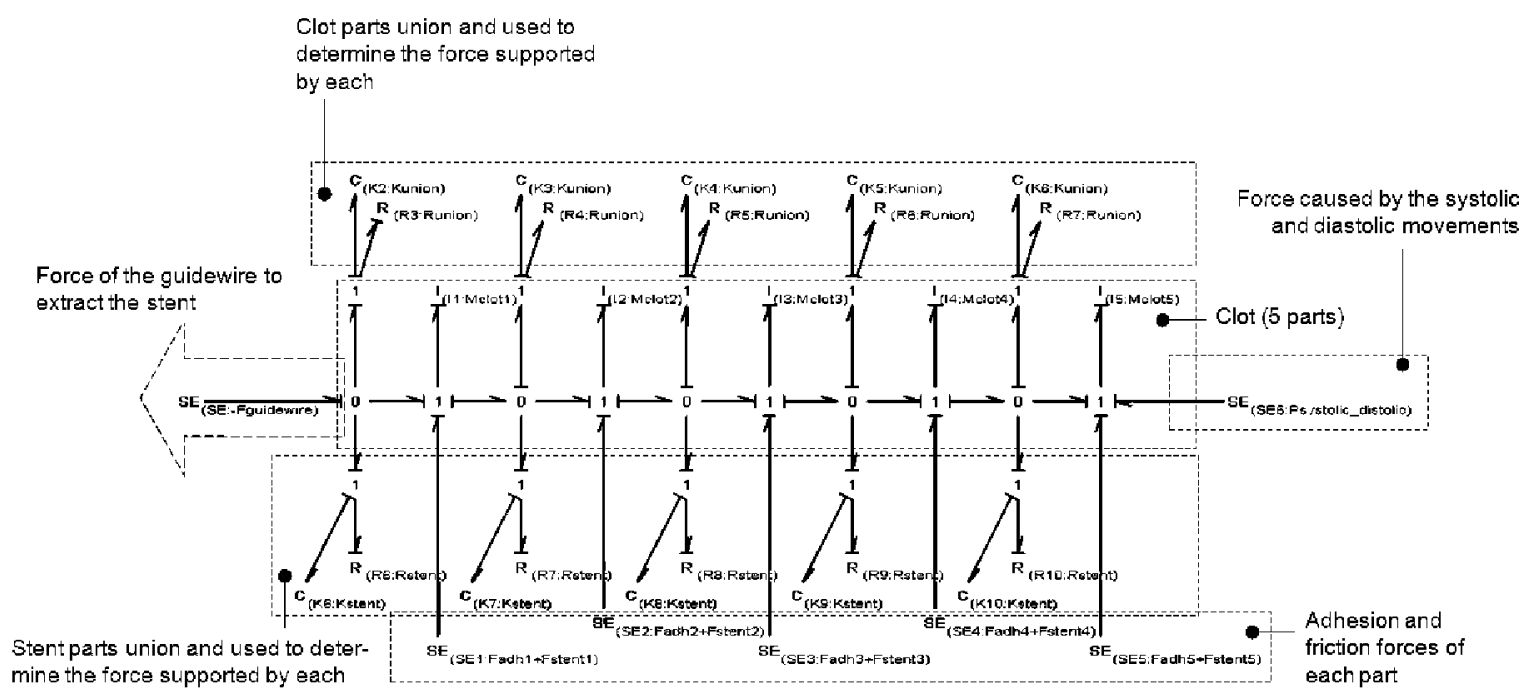

Figure 6. Model of blood clot-artery, guidewire and stent interaction

\section{RESULTS}

The aim of this simulation is to determine the time required for the extraction of a blood clot with a selfexpandable stent, whose rigidity is variable due to the used nitinol material.

To do this, by varying the values of the force of the guidewire, the movement of each partition of the clot and the time required for its extraction are measured, thereby obtaining the optimum force. Such modelling could potentially help us in the prevention of excessive forces, causing the rupture of the blood clot, if a typical stent were used in the smallest arteries.

To carry out the model validation, we use values for the parameters (in the simulation) listed in the following table.

\begin{tabular}{c|c}
\multicolumn{2}{c}{ TABLE I. Parameter Values } \\
Force & {$\left[4.09 \cdot 10^{-4}, 0.5\right] \mathrm{N}$} \\
\hline Blood Density $(\rho)$ & $1060 \mathrm{Kg} / \mathrm{m}^{3}$ \\
\hline Stent length $(\mathrm{L})$ & $0.02 \mathrm{~m}$ \\
\hline Artery diameter & $3.0 \mathrm{~mm}$ \\
\hline Blood Viscosity $(\eta)$ & $0.0035 \mathrm{~kg} / \mathrm{m} \cdot \mathrm{s}$ \\
\hline $\mathrm{K}_{\text {union }}$ & $1.91 \mathrm{~N} / \mathrm{m}$ \\
\hline $\mathrm{R}_{\text {union }}$ & $0.035 \mathrm{~kg} / \mathrm{m} \cdot \mathrm{s}$ \\
\hline Stent diameter $\left(\mathrm{D}_{0}\right)$ & $2.0,3.0,5.0 \cdot 10^{-3} \mathrm{~m}$ \\
\hline Clot length $(\mathrm{Lc})$ & {$[20,30] \cdot 10^{-3} \mathrm{~m}$} \\
\hline Percentage of platelets $(\mathrm{fp})$ & 0.96 \\
\hline Mean Platelet Area $(\mathrm{MPA})$ & $5.31 \cdot 10^{-12} \mathrm{~m}^{2}$ \\
\hline F $_{\text {adhesion platelet }}$ & $32 \cdot 10^{-9} \mathrm{~N}$ \\
\hline Occlusion & $100 \%$ \\
\hline Number of wires $(\mathrm{n})$ & 8 \\
\hline Initial pitch angle $\left(\beta_{0}\right)$ & $45^{\circ}$ \\
\hline Stent Young Modulus $(\mathrm{E})$ & $221000 \mathrm{MN} / \mathrm{m}^{2}$ \\
\hline Stent Shear Modulus $(\mathrm{G})$ & $81000 \mathrm{MN} / \mathrm{m}^{2}$ \\
\hline Wire diameter & $0.04 \mathrm{~mm}$
\end{tabular}

The parameters that define almost completely the elasticplastic behavior of the clot and its resistance to breaking are the constants $\mathrm{K}_{\text {union }}$ and $\mathrm{R}_{\text {union }}$ of the spring-damper systems in parallel that are among the partitions of the clot that characterize the clot in the stretch mode, when it suffers the suction but not yet detached from the wall.

To find the value of the $K_{\text {uпіо }}$ parameter, Savushkin [15] analyzes the stiffness of the clot and the breaking strength. We considered that the values are valid, due to the fact that the parameters of the experiments described fall within our range, and therefore we can assume that $\mathrm{K}_{\text {union }}=3.41 \pm 1.5$ $\mathrm{N} / \mathrm{m}$.

Concerning with the $\mathrm{R}_{\text {union }}$ value, Pennati [16] considers some useful parameters. In that work, values of the viscosity of the blood appear for the clot that they use in their model; taking into account the viscosity of the clot, we can assume that $\mathrm{R}_{\text {union }}=0.035 \mathrm{~kg} / \mathrm{m} \cdot \mathrm{s}$.

In the model simulated in this section, we take a blood clot of $3 \mathrm{~mm}$ of diameter and $2 \mathrm{~cm}$ of longitude. The existence of different partitions in the clot and also in the stent makes the extraction progressive with increasing time.

The parameters used have been taken to be fairly typical values so as to try to formulate a model that is as general as possible. The simulation results show that the greater rigidity of the clot, the shorter the extraction time. This factor is also related to the viscosity and composition of the clot that will vary in each case and patient. Therefore, for this study we have provided the critical values possible, by taking the situation where the clot is composed of $96 \%$ platelets. This gives rise to a fairly high bond strength - this could be reduced for other instances, but we use it here to give us an idea of the maximum pressure needed.

We also use a progressive force at the beginning of the experiment during $0.5 \mathrm{sec}$. and then it remains constant, focusing in the study on the required time for each partition to detach. Additionally, the variation of the radial and longitudinal stiffness value for the stent from this deformation has also been implemented. 
The following figures show the time that each partition takes to move, and the manner in which they fall off one to one as time increases.

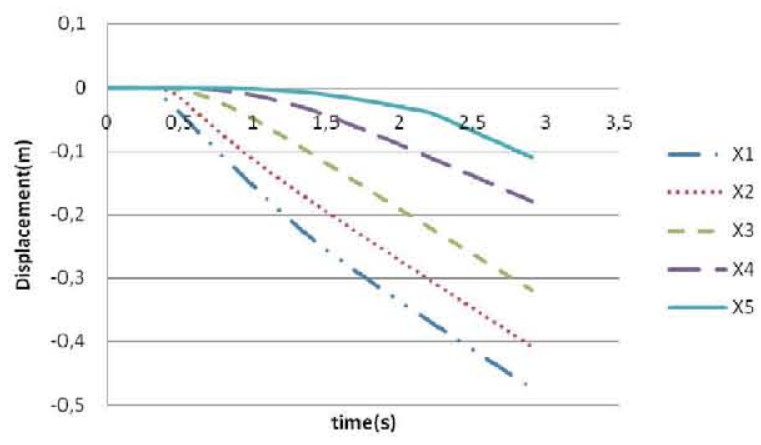

Figure 7. Blood clot partitions displacement.

In the following figure, we observe the variation of the longitudinal stiffness increase when it is pulled from the initial status into the artery, according with eq. (7).

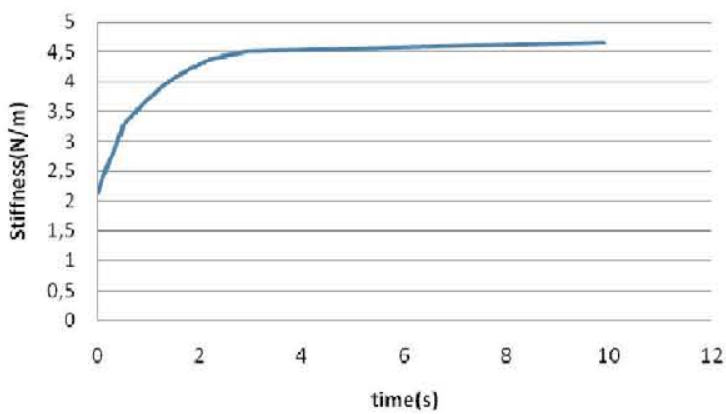

Figure 8. Results of the variation of the longitudinal stiffness.

As we can see in figure 8 , as time increases, the stiffness becomes greater, and the greater is the deformation suffered by the stent in accordance with figure 7 .

\section{CONCLUSIONS AND FUTURE WORKS}

The work presented in this paper incorporated a blood clot model based in five parts, which makes it more realistic that previous models done by the authors. Additionally, a stent system has been inserted, making it possible to simulate different situations. Many previous studies analyze similar models by using Finite Element Analysis, where it is often necessary to reproduce the full which usually requires a lot of computer simulation time.

Our study has demonstrated that the Bond Graph technique is very useful in representing different simulation conditions, making it possible to incorporate different parameters in a very effective straight forward manner. It is now being implemented to model and simulate a combination of the GPTAD and a self-expandable stent to improve the blood clots extraction process.

\section{REFERENCES}

[1] O'Riordan, M. 2004. Stenting and Angioplasty with Protection in Patients at High Risk for Endarterectomy. New England Journal of Medicine.

[2] Cerebrovascular Interventional Radiology: Mech. Thromb. in the acute Stroke Setting. Upcoming Advancements. http://bme240.eng.uci.edu/students/10s/vatanpos/upcoming.htm . Last accessed September $20^{\text {th }} 2011$.

[3] EV3 manufacturer. < http://www.ev3.net/assets/007/5788.pdf>. Last accessed July $03^{\text {rd }} 2011$.

[4] Karnopp, D.C., Margolis, D.L. and Rosemberg, R.C. 1990. "System Dynamics: A Unified Approach". John Wiley \& Sons, Inc., Second edition.

[5] Zadpoor, A.A., Arshi, A.R., Nikooyan, A.A. 2005. A bond graph approach to the modelling of fluid-solid interaction in cardiovascular system's pulsatile flow. 27th Annual International Conference of the IEEE in Medicine and Biology Society (EMBC05), Sept 1st - 4th, 2005, Shanghai, China.

[6] Romero, G., Higuera, I., Martinez, M.L., Pearce, G., Perkinson, N.D., Roffe, C., Wong, J. 2010. Computational modelling and optimization of a new Thrombectomy Device for the extraction of blood clots. Chapter book "Advances in Computational Biology", inside serie "Advances in Experimental Medicine and Biology, AEMB". Ed. Springer. Vol 680(6), pp 627-633.

[7] Romero, G., Higuera, I., Martinez, M.L., Pearce, G., Perkinson, N.D. 2010. Simulation of the GPTAD applied to the removal of blood clots that arise during Peripheral Vascular Disease. 1st International Conference on Intelligent Systems, Modelling and Simulation (ISMS'2010), pp 164-169. Liverpool, UK. January 2010.

[8] Romero, G., Martinez, M.L., Felez, J., Pearce, G., Perkinson, N.D. 2011; "Applicability of the GP Device to the Circle of Willis Arteries by Using a Mathematical Model". 13th International Conference on Modelling and Simulation - UkSim-AMSS 2011, pp. 48-53. Cambridge, UK. March 2011.

[9] Margolis, D. 1979. Bond graph fluid line models for inclusion with dynamic systems simulations". Journal of the Franklin Institute $308(3), 1979$, pp 255-268.

[10] Flannery, C. J.. 2005. PhD. Thesis, Thrombus Formation under High Shear in Arterial Stenotic Flow. Georgia Institute of Technology. USA.

[11] Jedwab, M. R., Clerc, C. O. 1993. A study of the geometrical an mechanical properties of a selfexpanding metallic stent - theory and experiment. Journal of Applied Biomaterials.

[12] Conti, P. 2006. PhD. Thesis, Finite Element Analysis of selfexpanding braided wirestent. Universita' degli Studi di Pavia and Ghent University.

[13] Wahl, A.M. 1963. Mechanical Springs, $2^{\text {nd }}$ edition. McGraw-Hill book Company.

[14] Loo, K.V. 2005. PhD. Thesis, Modellering en simulatie van zelfexpandeerbare draadstents. Laboratorium voor modelonderzoek. Ghent University.

[15] Savushkin, A.V.. 2003. Clots of Blood Plasma. Journal of Engineering Physics and Thermophysics, 76(3).

[16] Pennati, G., Balossino, R., Dubini, G., Migliavacca, F. 2010. Numerical Simulation of Thrombus Aspiration in Two Realistic Models of Catheter. Artificial Organs 34(4), pp. 301-310. 